\title{
Impulsive Predator-Prey Dynamic Systems with Beddington-DeAngelis Type Functional Response on the Unification of Discrete and Continuous Systems
}

\author{
Ayşe Feza Güvenilir ${ }^{1}$, Billur Kaymakçalan², Neslihan Nesliye Pelen ${ }^{3}$ \\ ${ }^{1}$ Department of Mathematics, Ankara University, Ankara, Turkey \\ ${ }^{2}$ Department of Mathematics, Çankaya University, Ankara, Turkey \\ ${ }^{3}$ Department of Mathematics, Ondokuz Mays University, Samsun, Turkey \\ Email: guvenili@science.ankara.edu.tr, billurkaymakcalan@gmail.com, nesliyeaykir@gmail.com
}

Received 27 July 2015; accepted 25 August 2015; published 28 August 2015

Copyright (C) 2015 by authors and Scientific Research Publishing Inc.

This work is licensed under the Creative Commons Attribution International License (CC BY). http://creativecommons.org/licenses/by/4.0/

(c) (7) Open Access

\begin{abstract}
In this study, the impulsive predator-prey dynamic systems on time scales calculus are studied. When the system has periodic solution is investigated, and three different conditions have been found, which are necessary for the periodic solution of the predator-prey dynamic systems with Beddington-DeAngelis type functional response. For this study the main tools are time scales calculus and coincidence degree theory. Also the findings are beneficial for continuous case, discrete case and the unification of both these cases. Additionally, unification of continuous and discrete case is a good example for the modeling of the life cycle of insects.
\end{abstract}

\section{Keywords}

Time Scales Calculus, Predator-Prey Dynamic Systems, Periodic Solutions, Coincidence Degree Theory, Beddington-DeAngelis Type Functional Response

\section{Introduction}

The relationships between species and the outer environment, and the connections between different species are the description of the predator-prey dynamic systems which is the subject of mathematical ecology in biomathematics. Various types of functional responses in predator-prey dynamic system such as Monod-type, semi-ratiodependent and Holling-type have been studied. [1] is an example for the study about Holling-type functional re- 
sponse. In this paper, we consider the predator-prey system with Beddington DeAngelis type functional response and impulses. This type of functional response first appeared in [2] and [3]. At low densities this type of functional response can avoid some of the singular behavior of ratio-dependent models. Also predator feeding can be described much better over a range of predator-prey abundances by using this functional response.

In a periodic environment, significant problem in population growth model is the global existence and stability of a positive periodic solution. This plays a similar role as a globally stable equilibrium in an autonomous model. Therefore, it is important to consider under which conditions the resulting periodic nonautonomous system would have a positive periodic solution that is globally asymptotically stable. For nonautonomous case there are many studies about the existence of periodic solutions of predator-prey systems in continuous and discrete models based on the coincidence theory such as [4]-[12].

Impulsive dynamic systems are also important in this study and we try to give some information about this area. Impulsive differential equations are used for describing systems with short-term perturbations. Its theory is explained in [13]-[15] for continuous case and also for discerete case there are some studies such as [16]. Impulsive differential equations are widely used in many different areas such as physics, ecology, and pest control. Most of them use impulses at fixed time such as [17] [18]. By using constant functions, some properties of the solution of predator-prey system with Beddington-DeAnglis type functional response and impulse impact are studied in [19] for continuous case.

In this study unification of continuous and discrete analysis is also significant. To unify the study of differential and difference equations, the theory of Time Scales Calculus is initiated by Stephan Hilger. In [20] [21], unification of the existence of periodic solutions of population models modelled by ordinary differential equations and their discrete analogues in form of difference equations, and extension of these results to more general time scales are studied.

The unification of continuous and discrete case is a good example for the modeling of the life cycle of insects. Most of the insects have a continuous life cycle during the warm months of the year and die out in the cold months of the year, and in that period their eggs are incubating or dormant. These incubating eggs become new individuals of the new warm season. Since insects have such a continuous and discrete life cycle, we can see the importance of models obtained by the time scales calculus for the species that have unusual life cycle. Therefore, in this paper we try to generalize periodic solutions of predator-prey dynamic systems with Beddington-DeAnglis type functional response and impulse to general time scales.

\section{Preliminaries}

Below informations are from [20]. Let $X, Z$ be normed vector spaces, $L: D o m L \subset X \rightarrow Z$ be a linear mapping, $N: X \rightarrow Z$ be a continuous mapping. The mapping $L$ will be called a Fredholm mapping of index zero if $\operatorname{dimKer} L=$ codimImL $<+\infty$ and $\operatorname{ImL}$ is closed in $Z$. If $L$ is a Fredholm mapping of index zero and there exist continuous projections $P: X \rightarrow X$ and $Q: Z \rightarrow Z$ such that $\operatorname{Im} P=\operatorname{Ker} L, \operatorname{Im} L=\operatorname{Ker} Q=\operatorname{Im}(I-Q)$, then it follows that $\left.L\right|_{\text {DomL } \cap \text { KerP }}:(I-P) X \rightarrow \operatorname{ImL}$ is invertible. We denote the inverse of that map by $K_{P}$. If $\Omega$ is an open bounded subset of $X$, the mapping $N$ will be called $L$-compact on $\Omega$ if $Q N(\Omega)$ is bounded and $K_{P}(I-Q) N: \Omega \rightarrow X$ is compact. Since ImQ is isomorphic to KerL, there exists an isomorphism $J: \operatorname{ImQ} \rightarrow \operatorname{KerL}$.

The above informations are important for the Continuation Theorem that we give below.

Theorem 1. (Continuation Theorem). Let L be a Fredholm mapping of index zero and $N$ be L-compact on $\Omega$. Suppose

(a) For each $\lambda \in(0,1)$, every solution $\mathrm{z}$ of $\mathrm{Lz}=\lambda \mathrm{Nz}$ is such that $\mathrm{z} \notin \delta \Omega$;

(b) $Q N z \neq 0$ for each $z \in \delta \Omega \cap K e r L$ and the Brouwer degree deg $\{J Q N, \delta \Omega \cap K e r L, 0\} \neq 0$. Then the operator equation $\mathrm{Lz}=\mathrm{Nz}$ has at least one solution lying in DomL $\cap \delta \Omega$.

We will also give the following lemma, which is essential for this paper.

Lemma 1. Let $t_{1}, t_{2} \in[0, \omega]$ and $t \in \mathbb{T}$. If $g: T \rightarrow R$ is $\omega$-periodic, then

$$
g(t) \leq g\left(t_{1}\right)+\int_{0}^{\omega}\left|g^{\Delta}(s)\right| \Delta s \quad \text { and } \quad g(t) \geq g\left(t_{2}\right)-\int_{0}^{\omega}\left|g^{\Delta}(s)\right| \Delta s .
$$

\section{Main Result}

The equation that we investigate is: 


$$
\begin{aligned}
& x^{\Delta}(t)=a(t)-b(t) \exp (x(t))-\frac{c(t) \exp (y(t))}{\alpha(t)+\beta(t) \exp (x(t))+m(t) \exp (y(t))}, t \neq t_{k} \\
& y^{\Delta}(t)=-d(t)+\frac{f(t) \exp (x(t))}{\alpha(t)+\beta(t) \exp (x(t))+m(t) \exp (y(t))}, t \neq t_{k} \\
& \Delta x\left(t_{k}\right)=\ln \left(1+g_{k}\right) \\
& \Delta y\left(t_{k}\right)=\ln \left(p_{k}\right)
\end{aligned}
$$

$t_{k+q}=t_{k}+w, \quad a(t+w)=a(t), \quad b(t+w)=b(t), \quad c(t+w)=c(t), \quad d(t+w)=d(t), \quad f(t+w)=f(t)$, $\alpha(t+w)=\alpha(t), \beta(t+w)=\beta(t), \quad m(t+w)=m(t), \quad \forall k, 1>g_{k}>-1$, and $p_{k}>0$. Here $\mathbb{T}$ is periodic, i.e if $t \in \mathbb{T}$ then $t+w \in \mathbb{T}$, and $\int_{0}^{w} a(t) \Delta t>0, \int_{0}^{w} b(t) \Delta t>0, \quad \int_{0}^{w} d(t) \Delta t>0 \quad \beta^{l}=\min _{t \in[0, w]} \beta(t)$, $m^{l}=\min _{t \in[0, w]} m(t), \quad \beta^{u}=\max _{t \in[0, w]} \beta(t), m^{u}=\max _{t \in[0, w]} m(t), m(t)>0$ and $c(t), f(t)>0, b(t), \alpha(t) \geq 0$, $\beta(t)>0$. Each functions are from $C_{r d}(\mathbb{T}, \mathbb{R})$.

Lemma 2. If $\int_{0}^{w} a(t) \Delta t+\ln \prod_{i=1}^{q}\left(1+g_{i}\right)<0$ and $-\int_{0}^{w} d(t) \Delta t+\ln \prod_{i=1}^{q} p_{i}+\int_{0}^{w} \frac{f(t)}{\beta(t)} \Delta t<0$, then all positive solutions $(\exp (x(t)), \exp (y(t)))$ are tends to 0 as $t$ tends to infinity.

Proof. If we using the first equation of (1) we obtain,

$$
\exp (x(t)) \leq \exp (x(0)) \prod_{t i<t}\left(1+g_{i}\right) \exp \left(\int_{0}^{t} a(s) \Delta s\right)
$$

Since $\int_{0}^{w} a(t) \Delta t+\ln \prod_{i=1}^{q}\left(1+g_{i}\right)<0$. Hence $\lim _{t \rightarrow \infty} \exp (x(t))=0$.

Similarly $\lim _{t \rightarrow \infty} \exp (y(t))=0$.

Theorem 2. In addition to conditions on coefficient functions

If

$$
\int_{0}^{w} a(t) \Delta t+\ln \prod_{i=1}^{q}\left(1+g_{i}\right)-\int_{0}^{w} \frac{c(t)}{m(t)} \Delta t>0
$$

and

$$
\begin{aligned}
& \left(\frac{\int_{0}^{w} a(t) \Delta t+\ln \prod_{i=1}^{q}\left(1+g_{i}\right)-\int_{0}^{w} \frac{c(t)}{m(t)} \Delta t}{\int_{0}^{w} b(t) \Delta t}\right) \exp \left[-\left(\int_{0}^{w}|a(t)| \Delta t+\int_{0}^{w} a(t) \Delta t+\ln \prod_{i=1}^{q}\left(1+g_{i}\right)\right)\right] \\
& \cdot\left(\int_{0}^{w} f(t) \Delta t-\beta^{u}\left(\int_{0}^{w} d(t) \Delta t-\ln \prod_{i=1}^{q}\left(p_{i}\right)\right)\right)-\alpha^{u}\left(\int_{0}^{w} d(t) \Delta t-\ln \prod_{i=1}^{q}\left(p_{i}\right)\right)>0
\end{aligned}
$$

then there exist at least a w-periodic solution.

Proof. $\quad X:=\left\{\left[\begin{array}{l}u \\ v\end{array}\right] \in P C\left(\mathbb{T}, \mathbb{R}^{2}\right): u(t+w)=u(t), v(t+w)=v(t)\right\}$ with the norm:

$$
\left\|\left[\begin{array}{l}
u \\
v
\end{array}\right]\right\|=\sup _{t \in[0, w]_{\mathbb{T}}}(|u(t)|,|v(t)|)
$$

and

$$
Y:=\left\{\left[\left[\begin{array}{l}
u \\
v
\end{array}\right],\left[\begin{array}{l}
a_{1} \\
b_{1}
\end{array}\right], \cdots,\left[\begin{array}{l}
a_{q} \\
b_{q}
\end{array}\right]\right] \in P C\left(\mathbb{T}, \mathbb{R}^{2}\right) \times\left(\mathbb{R}^{2}\right)^{q}, u(t+w)=u(t), v(t+w)=v(t)\right\}
$$


with the norm:

$$
\left\|\left[\left[\begin{array}{l}
u \\
v
\end{array}\right],\left[\begin{array}{l}
a_{1} \\
b_{1}
\end{array}\right], \cdots,\left[\begin{array}{l}
a_{q} \\
b_{q}
\end{array}\right]\right]\right\|=\sup _{t \in[0, w]_{\mathbb{T}}}\left(\left\|\left[\begin{array}{l}
u \\
v
\end{array}\right]\right\|,\left\|\left[\begin{array}{l}
a_{1} \\
b_{1}
\end{array}\right]\right\|, \cdots,\left\|\left[\begin{array}{l}
a_{q} \\
b_{q}
\end{array}\right]\right\|\right) .
$$

Let us define the mappings $L$ and $N$ by $L: D o m L \subset X \rightarrow Y$ such that

$$
L\left(\left[\begin{array}{l}
u \\
v
\end{array}\right]\right)=\left(\left[\begin{array}{l}
u^{\Delta} \\
v^{\Delta}
\end{array}\right],\left[\begin{array}{l}
\Delta u\left(t_{1}\right) \\
\Delta v\left(t_{1}\right)
\end{array}\right], \cdots,\left[\begin{array}{l}
\Delta u\left(t_{q}\right) \\
\Delta v\left(t_{q}\right)
\end{array}\right]\right)
$$

and $N: X \rightarrow Y$ such that

$$
N\left(\left[\begin{array}{l}
u \\
v
\end{array}\right]\right)=\left(\left[\begin{array}{c}
a(t)-b(t) \exp (u(t))-\frac{c(t) \exp (v(t))}{\alpha(t)+\beta(t) \exp (u(t))+m(t) \exp (v(t))} \\
-d(t)+\frac{f(t) \exp (u(t))}{\alpha(t)+\beta(t) \exp (u(t))+m(t) \exp (v(t))}
\end{array}\right],\left[\begin{array}{c}
\ln \left(1+g_{1}\right) \\
\ln \left(p_{1}\right)
\end{array}\right], \ldots,\left[\begin{array}{c}
\ln \left(1+g_{q}\right) \\
\ln \left(p_{q}\right)
\end{array}\right]\right) .
$$

Then $\operatorname{KerL}=\left\{\left[\begin{array}{l}u \\ v\end{array}\right]:\left[\begin{array}{l}u \\ v\end{array}\right]=\left[\begin{array}{l}c_{1} \\ c_{2}\end{array}\right]\right\}, \quad c_{1}$ and $c_{2}$ are constants.

$$
\operatorname{ImL}=\left\{\left[\left[\begin{array}{l}
u \\
v
\end{array}\right],\left[\begin{array}{l}
a_{1} \\
b_{1}
\end{array}\right], \cdots,\left[\begin{array}{l}
a_{q} \\
b_{q}
\end{array}\right]\right]:\left[\begin{array}{l}
\int_{0}^{w} u(s) \Delta s+\sum_{i=1}^{q} a_{i} \\
\int_{0}^{w} v(s) \Delta s+\sum_{i=1}^{q} b_{i}
\end{array}\right]=\left[\begin{array}{l}
0 \\
0
\end{array}\right]\right\} .
$$

$\operatorname{ImL}$ is closed in $Y$ and $\operatorname{dimKer} L=\operatorname{codim} \operatorname{Im} L=2$, therefore $L$ is a Fredholm mapping of index zero.

There exist continuous projectors $P: X \rightarrow X$ and $Q: Y \rightarrow Y$ such that

$$
P\left(\left[\begin{array}{l}
u \\
v
\end{array}\right]\right)=\frac{1}{\operatorname{mes}(w))}\left[\begin{array}{c}
\int_{0}^{w} u(s) \Delta s \\
\int_{0}^{w} v(s) \Delta s
\end{array}\right]
$$

and

$$
Q\left(\left[\begin{array}{l}
u \\
v
\end{array}\right],\left[\begin{array}{l}
a_{1} \\
b_{1}
\end{array}\right], \cdots,\left[\begin{array}{l}
a_{q} \\
b_{q}
\end{array}\right]\right)=\frac{1}{\operatorname{mes}(w)}\left(\left[\begin{array}{c}
\int_{0}^{w} u(s) \Delta s+\sum_{i=1}^{q} a_{i} \\
\int_{0}^{w} v(s) \Delta s+\sum_{i=1}^{q} b_{i}
\end{array}\right],\left[\begin{array}{l}
0 \\
0
\end{array}\right], \cdots,\left[\begin{array}{l}
0 \\
0
\end{array}\right]\right),
$$

where mes $(t)=\int_{0}^{t} 1 \Delta t$.

The generalized inverse $K_{P}=\operatorname{ImL} \rightarrow \operatorname{DomL} \subset \operatorname{Ker} P$ is given,

$$
\begin{aligned}
& K_{P}\left(\left[\begin{array}{l}
u \\
v
\end{array}\right],\left[\begin{array}{l}
a_{1} \\
b_{1}
\end{array}\right], \cdots,\left[\begin{array}{l}
a_{q} \\
b_{q}
\end{array}\right]\right)=\left[\begin{array}{l}
\int_{0}^{t} u(s) \Delta s+\sum_{t>t_{i}} a_{i}-\frac{1}{m e s(w)} \int_{0}^{w} \int_{0}^{t} u(s) \Delta s \Delta t-\sum_{i=1}^{q} a_{i}+\frac{1}{m e s(w)} \sum_{i=1}^{q} a_{i} \operatorname{mes}\left(t_{i}\right) \\
\int_{0}^{t} v(s) \Delta s+\sum_{t>t_{i}} b_{i}-\frac{1}{m e s(w)} \int_{0}^{w} \int_{0}^{t} v(s) \Delta s \Delta t-\sum_{i=1}^{q} b_{i}+\frac{1}{m e s(w)} \sum_{i=1}^{q} b_{i} m e s\left(t_{i}\right)
\end{array}\right] . \\
& Q N\left(\left[\begin{array}{l}
u \\
v
\end{array}\right]\right)=\frac{1}{m e s(w)}\left(\left[\begin{array}{c}
\int_{0}^{w} a(s)-b(s) \exp (u(s))-\frac{c(s) \exp (v(s))}{\alpha(s)+\beta(s) \exp (u(s))+m(s) \exp (v(s))} \Delta s+\ln \prod_{i=1}^{q}\left(1+g_{i}\right) \\
\int_{0}^{w}-d(s)+\frac{f(s) \exp (u(s))}{\alpha(s)+\beta(s) \exp (u(s))+m(s) \exp (v(s))} \Delta s+\ln \prod_{i=1}^{q}\left(p_{i}\right)
\end{array}\right], \cdots,\left[\begin{array}{l}
0 \\
0
\end{array}\right]\right)
\end{aligned}
$$


Let

$$
\begin{aligned}
& a(t)-b(t) \exp (u(t))-\frac{c(t) \exp (v(t))}{\alpha(t)+\beta(t) \exp (u(t))+m(t) \exp (v(t))}=N_{1}, \\
& -d(t)+\frac{f(t) \exp (u(t))}{\alpha(t)+\beta(t) \exp (u(t))+m(t) \exp (v(t))}=N_{2}, \\
& \frac{1}{m e s(w)} \int_{0}^{w} a(s)-b(s) \exp (u(s))-\frac{c(s) \exp (v(s))}{\alpha(s)+\beta(s) \exp (u(s))+m(s) \exp (v(s))} \Delta s=\bar{N}_{1},
\end{aligned}
$$

and

$$
\begin{aligned}
& \frac{1}{\operatorname{mes}(w)} \int_{0}^{w}-d(s)+\frac{f(s) \exp (u(s))}{\alpha(s)+\beta(s) \exp (u(s))+m(s) \exp (v(s))} \Delta s=\bar{N}_{2} . \\
& K_{P}(I-Q) N\left(\left[\begin{array}{l}
u \\
v
\end{array}\right]\right)=K_{P}\left(\left[\begin{array}{c}
N_{1}-\bar{N}_{1} \\
N_{2}-\bar{N}_{2}
\end{array}\right],\left[\begin{array}{c}
\ln \left(1+g_{1}\right) \\
\ln \left(p_{1}\right)
\end{array}\right], \cdots,\left[\begin{array}{c}
\ln \left(1+g_{q}\right) \\
\ln \left(p_{q}\right)
\end{array}\right]\right) \\
& =\left[\begin{array}{c}
\int_{0}^{t} N_{1}(s)-\bar{N}_{1}(s) \Delta s+\ln \prod_{t>t_{i}}\left(1+g_{i}\right)-\frac{1}{m e s(w)} \int_{0}^{w} \int_{0}^{t} N_{1}(s)-\bar{N}_{1}(s) \Delta s \Delta t-\ln \prod_{i=1}^{q}\left(1+g_{i}\right)+\frac{1}{m e s(w)} \sum_{i=1}^{q} \ln \left(1+g_{i}\right) t_{i} \\
\int_{0}^{t} N_{2}(s)-\bar{N}_{2}(s) \Delta s+\ln \prod_{t>t_{i}} p_{i}-\frac{1}{\operatorname{mes}(w)} \int_{0}^{w} \int_{0}^{t} N_{2}(s)-\bar{N}_{2}(s) \Delta s \Delta t-\ln \prod_{i=1}^{q} p_{i}+\frac{1}{\operatorname{mes}(w)} \sum_{i=1}^{q} \ln \left(p_{i}\right) t_{i}
\end{array}\right] .
\end{aligned}
$$

Clearly, $Q N$ and $K_{P}(I-Q) N$ are continuous. Since $X$ and $Y$ are Banach spaces, then by using ArzelaAscoli theorem we can find $\bar{K}_{P}(I-Q) N(\bar{\Omega})$ is compact for any open bounded set $\Omega \subset X$. Additionally, $Q N(\bar{\Omega})$ is bounded. Thus, $N$ is $L$-compact on $\bar{\Omega}$ with any open bounded set $\Omega \subset X$.

To apply the continuation theorem we investigate the below operator equation.

$$
\begin{aligned}
& x^{\Delta}(t)=\lambda\left[a(t)-b(t) \exp (x(t))-\frac{c(t) \exp (y(t))}{\alpha(t)+\beta(t) \exp (x(t))+m(t) \exp (y(t))}\right], t \neq t_{k} \\
& y^{\Delta}(t)=\lambda\left[-d(t)+\frac{f(t) \exp (x(t))}{\alpha(t)+\beta(t) \exp (x(t))+m(t) \exp (y(t))}\right], t \neq t_{k} \\
& \Delta x\left(t_{k}\right)=\lambda \ln \left(1+g_{k}\right) \\
& \Delta y\left(t_{k}\right)=\lambda \ln \left(p_{k}\right)
\end{aligned}
$$

Let $\left[\begin{array}{l}x \\ y\end{array}\right] \in X$ be any solution of system (2). Integrating both sides of system (2) over the interval $[0, w]$ we obtain,

$$
\left\{\begin{array}{l}
\int_{0}^{w} a(t) \Delta t+\ln \prod_{i=1}^{q}\left(1+g_{i}\right)=\int_{0}^{w} b(t) \exp (x(t))+\frac{c(t) \exp (y(t))}{\alpha(t)+\beta(t) \exp (x(t))+m(t) \exp (y(t))} \Delta t, \\
\int_{0}^{w} d(t) \Delta t-\ln \prod_{i=1}^{q}\left(p_{i}\right)=\int_{0}^{w} \frac{f(t) \exp (x(t))}{\alpha(t)+\beta(t) \exp (x(t))+m(t) \exp (y(t))} \Delta t
\end{array}\right.
$$

From (2) and (3) we get 


$$
\begin{aligned}
\int_{0}^{w}\left|x^{\Delta}(t)\right| \Delta t & \leq \lambda\left[\int_{0}^{w}|a(t)| \Delta t+\int_{0}^{w} b(t) \exp (x(t))+\frac{c(t) \exp (y(t))}{\alpha(t)+\beta(t) \exp (x(t))+m(t) \exp (y(t))} \Delta t\right] \\
& \leq \lambda\left[\int_{0}^{w}|a(t)| \Delta t+\int_{0}^{w} a(t) \Delta t+\ln \prod_{i=1}^{q}\left(1+g_{i}\right)\right] \leq M_{1} ;
\end{aligned}
$$

where $M_{1}:=\int_{0}^{w}|a(t)| \Delta t+\int_{0}^{w} a(t) \Delta t+\ln \prod_{i=1}^{q}\left(1+g_{i}\right)$.

$$
\begin{aligned}
\int_{0}^{w}\left|y^{\Delta}(t)\right| \Delta t & \leq \lambda\left[\int_{0}^{w}|d(t)| \Delta t+\int_{0}^{w} \frac{f(t) \exp (x(t))}{\alpha(t)+\beta(t) \exp (x(t))+m(t) \exp (y(t))} \Delta t\right] \\
& \leq \lambda\left[\int_{0}^{w}|d(t)| \Delta t+\int_{0}^{w} d(t) \Delta t-\ln \prod_{i=1}^{q} p_{i}\right] \leq M_{2}
\end{aligned}
$$

where $M_{2}:=\int_{0}^{w}|d(t)| \Delta t+\int_{0}^{w} d(t) \Delta t-\ln \prod_{i=1}^{q} p_{i}$.

Note that since $\left[\begin{array}{l}x \\ y\end{array}\right] \in X$ and there are q impulses which are constant, then there exist $\eta_{i}, \xi_{i}, i=1,2$ such that

$$
\begin{aligned}
& x\left(\xi_{1}\right)=\min \left\{\inf _{t \in\left[0, t_{1}\right]} x(t), \inf _{t \in\left(t_{1}, t_{2}\right]} x(t), \cdots, \inf _{t \in\left[t_{q}, w\right]} x(t)\right\} \\
& x\left(\eta_{1}\right)=\max \left\{\sup _{t \in\left[0, t_{1}\right]} x(t), \sup _{t \in\left(t_{1}, t_{2}\right]} x(t), \cdots, \sup _{t \in\left(t_{q}, w\right]} x(t)\right\} \\
& y\left(\xi_{2}\right)=\min \left\{\inf _{t \in\left[0, t_{1}\right]} y(t), \inf _{t \in\left[t_{1}, t_{2}\right]} y(t), \cdots, \inf _{t \in\left[t_{q}, w\right]} y(t)\right\} \\
& y\left(\eta_{2}\right)=\max \left\{\sup _{t \in\left[0, t_{1}\right]} y(t), \sup _{t \in\left[t_{1}, t_{2}\right]} y(t), \cdots, \sup _{t \in\left[t_{q}, w\right]} y(t)\right\}
\end{aligned}
$$

By the second equation of (3) and (6) and the first assumption of Theorem 2, we have

$$
\begin{aligned}
\int_{0}^{w} a(t) \Delta t+\ln \prod_{i=1}^{q}\left(1+g_{i}\right) & \leq \int_{0}^{w}\left[b(t) \exp \left(x\left(\eta_{1}\right)\right)+\frac{c(t)}{m(t)}\right] \Delta t \\
& =\exp \left(x\left(\eta_{1}\right)\right) \int_{0}^{w} b(t) \Delta t+\int_{0}^{w} \frac{c(t)}{m(t)} \Delta t
\end{aligned}
$$

and $x\left(\eta_{1}\right) \geq l_{1} ;$ where $l_{1}:=\ln \left(\frac{\int_{0}^{w} a(t) \Delta t+\ln \prod_{i=1}^{q}\left(1+g_{i}\right)-\int_{0}^{w} \frac{c(t)}{m(t)} \Delta t}{\int_{0}^{w} b(t) \Delta t}\right)$.

Using the second inequality in Lemma 1 we have

$$
\begin{aligned}
x(t) & \geq x\left(\eta_{1}\right)-\int_{0}^{w}\left|x^{\Delta}(t)\right| \Delta t \\
& \geq x\left(\eta_{1}\right)-\left(\int_{0}^{w}|a(t)| \Delta t+\int_{0}^{w} a(t) \Delta t+\ln \prod_{i=1}^{q}\left(1+g_{i}\right)\right) \\
& \geq H_{1}:=l_{1}-M_{1} .
\end{aligned}
$$

By the first equation of (3) and (6) we get $x\left(\xi_{1}\right) \leq l_{2}$, where 


$$
l_{2}:=\ln \left(\frac{\int_{0}^{w} a(t) \Delta t+\ln \prod_{i=1}^{q}\left(1+g_{i}\right)}{\int_{0}^{w} b(t) \Delta t}\right) .
$$

using the first inequality in Lemma 1 and (4), we have

$$
\begin{aligned}
x(t) & \leq x\left(\xi_{1}\right)+\int_{0}^{w}\left|x^{\Delta}(t)\right| \Delta t \\
& \leq x\left(\xi_{1}\right)+\left(\int_{0}^{w}|a(t)| \Delta t+\int_{0}^{w} a(t) \Delta t+\ln \prod_{i=1}^{q}\left(1+g_{i}\right)\right) \\
& \leq H_{2}:=l_{2}+M_{1} .
\end{aligned}
$$

By (8) and (9) $\max _{t \in[0, w]}|x(t)| \leq B_{1}:=\max \left\{\left|H_{1}\right|,\left|H_{2}\right|\right\}$. Using (9), second equation of (3) and first equation of (7), we can derive that

$$
\begin{aligned}
& \int_{0}^{w} d(t) \Delta t-\ln \prod_{i=1}^{q}\left(p_{i}\right) \leq \int_{0}^{w} \frac{f(t) \exp (x(t))}{\beta^{l} \exp (x(t))+m^{l} \exp (y(t))} \Delta t \\
& \leq \int_{0}^{w} \frac{f(t) \mathrm{e}^{H_{2}}}{\beta^{l} \mathrm{e}^{H_{2}}+m^{l} \exp \left(y\left(\xi_{2}\right)\right)} \Delta t=\frac{\mathrm{e}^{H_{2}}}{\beta^{l} \mathrm{e}^{H_{2}}+m^{l} \exp \left(y\left(\xi_{2}\right)\right)} \int_{0}^{w} f(t) \Delta t
\end{aligned}
$$

Therefore

$$
\exp \left(y\left(\xi_{2}\right)\right) \leq \frac{1}{m^{l}}\left(\frac{\mathrm{e}^{H_{2}} \int_{0}^{w} f(t) \Delta t}{\int_{0}^{w} d(t) \Delta t-\ln \prod_{i=1}^{q}\left(p_{i}\right)}-\beta^{l} \mathrm{e}^{H_{2}}\right)
$$

By the assumption of the theorem we can show that

$$
\int_{0}^{w} f(t) \Delta t-\beta^{l}\left(\int_{0}^{w} d(t) \Delta t-\ln \prod_{i=1}^{q}\left(p_{i}\right)\right)>0 \text { and } y\left(\xi_{2}\right) \leq L_{1}
$$

where $L_{1}:=\ln \left(\frac{1}{m^{l}}\left(\frac{\mathrm{e}^{H_{2}} \int_{0}^{w} f(t) \Delta t}{\int_{0}^{w} d(t) \Delta t-\ln \prod_{i=1}^{q}\left(p_{i}\right)}-\beta^{l} \mathrm{e}^{H_{2}}\right)\right)$.

Hence, by using the first inequality in Lemma 1 and the second equation of (3),

$$
\begin{aligned}
y(t) & \leq y\left(\xi_{2}\right)+\int_{0}^{w}\left|y^{\Delta}(t)\right| \Delta t \\
& \leq y\left(\xi_{2}\right)+\left(\int_{0}^{w}|d(t)| \Delta t+\int_{0}^{w} d(t) \Delta t-\ln \prod_{i=1}^{q}\left(p_{i}\right)\right) \\
& \leq H_{3}:=L_{1}+M_{2} .
\end{aligned}
$$

We can also derive from the second equation of (3) that

$$
\begin{aligned}
& \int_{0}^{w} d(t) \Delta t-\ln \prod_{i=1}^{q}\left(p_{i}\right) \geq \int_{0}^{w} \frac{f(t) \exp (x(t))}{\alpha^{u}+\beta^{u} \exp (x(t))+m^{u} \exp (y(t))} \Delta t \\
& \geq \int_{0}^{w} \frac{f(t) \mathrm{e}^{H_{1}}}{\alpha^{u}+\beta^{u} \mathrm{e}^{H_{1}}+m^{u} \exp \left(y\left(\eta_{2}\right)\right)} \Delta t=\frac{\mathrm{e}^{H_{1}}}{\alpha^{u}+\beta^{u} \mathrm{e}^{H_{1}}+m^{u} \exp \left(y\left(\eta_{2}\right)\right)} \int_{0}^{w} f(t) \Delta t
\end{aligned}
$$




$$
\exp \left(y\left(\eta_{2}\right)\right) \geq \frac{1}{m^{u}}\left(\frac{\mathrm{e}^{H_{1}} \int_{0}^{w} f(t) \Delta t}{\int_{0}^{w} d(t) \Delta t-\ln \prod_{i=1}^{q}\left(p_{i}\right)}-\beta^{u} \mathrm{e}^{H_{1}}-\alpha^{u}\right) .
$$

Again using second assumption of Theorem 2 we obtain

$$
\mathrm{e}^{H_{1}}\left(\int_{0}^{w} f(t) \Delta t-\beta^{u}\left(\int_{0}^{w} d(t) \Delta t-\ln \prod_{i=1}^{q}\left(p_{i}\right)\right)\right)-\alpha^{u}\left(\int_{0}^{w} d(t) \Delta t-\ln \prod_{i=1}^{q}\left(p_{i}\right)\right)>0
$$

and $y\left(\eta_{2}\right) \geq L_{2}$ where $L_{2}:=\ln \left(\frac{1}{m^{u}}\left(\frac{\mathrm{e}^{H_{1}} \int_{0}^{w} f(t) \Delta t}{\int_{0}^{w} d(t) \Delta t-\ln \prod_{i=1}^{q}\left(p_{i}\right)}-\beta^{u} \mathrm{e}^{H_{1}}-\alpha^{u}\right)\right)$.

By using the second inequality in Lemma 1 and (5), we obtain

$$
\begin{aligned}
y(t) & \geq y\left(\eta_{2}\right)-\int_{0}^{w}\left|y^{\Delta}(t)\right| \Delta t \\
& \geq y\left(\eta_{2}\right)-\left(\int_{0}^{w}|d(t)| \Delta t+\int_{0}^{w} d(t) \Delta t-\ln \prod_{i=1}^{q}\left(p_{i}\right)\right) \\
& \geq H_{4}:=L_{2}-M_{2} .
\end{aligned}
$$

By (10) and (11) we have $\max _{t \in[0, w]}|y(t)| \leq B_{2}:=\max \left\{\left|H_{3}\right|,\left|H_{4}\right|\right\}$. Obviously, $B_{1}$ and $B_{2}$ are both independent of $\lambda$. Let $M=B_{1}+B_{2}+1$. Then $\max _{t \in[0, w]}\left\|\left[\begin{array}{l}x \\ y\end{array}\right]\right\|<M$. Let $\Omega=\left\{\left\|\left[\begin{array}{l}x \\ y\end{array}\right]\right\| \in X:\left\|\left[\begin{array}{l}x \\ y\end{array}\right]\right\|<M\right\}$ and $\Omega$ verifies the requirement (a) in Theorem 1 . When $\left[\begin{array}{l}x \\ y\end{array}\right] \in \operatorname{Ker} L \cap \partial \Omega,\left[\begin{array}{l}x \\ y\end{array}\right]$ is a constant with $\left\|\left[\begin{array}{l}x \\ y\end{array}\right]\right\|=M$, then

$$
\begin{aligned}
Q N\left(\left[\begin{array}{l}
x \\
y
\end{array}\right]\right) & =\left(\begin{array}{c}
{\left[\begin{array}{c}
\int_{0}^{w} a(s)-b(s) \exp (x)-\frac{c(s) \exp (y)}{\alpha(s)+\beta(s) \exp (x)+m(s) \exp (y)} \Delta s+\ln \prod_{i=1}^{q}\left(1+g_{i}\right) \\
\int_{0}^{w}-d(s)+\frac{f(s) \exp (x)}{\alpha(s)+\beta(s) \exp (x)+m(s) \exp (y)} \Delta s+\ln \prod_{i=1}^{q}\left(p_{i}\right)
\end{array}\right], \cdots,\left[\begin{array}{l}
0 \\
0
\end{array}\right]}
\end{array}\right] \\
& \neq\left(\left[\begin{array}{l}
0 \\
0
\end{array}\right], \cdots,\left[\begin{array}{l}
0 \\
0
\end{array}\right]\right) . \\
J Q N\left(\left[\begin{array}{l}
x \\
y
\end{array}\right]\right) & =\left[\begin{array}{c}
\int_{0}^{w} a(s)-b(s) \exp (x(s))-\frac{c(s) \exp (y(s))}{\alpha(s)+\beta(s) \exp (x(s))+m(s) \exp (y(s))} \Delta s+\ln \prod_{i=1}^{q}\left(1+g_{i}\right) \\
\int_{0}^{w}-d(s)+\frac{f(s) \exp (x(s))}{\alpha(s)+\beta(s) \exp (x(s))+m(s) \exp (y(s))} \Delta s+\ln \prod_{i=1}^{q}\left(p_{i}\right)
\end{array}\right],
\end{aligned}
$$

where $J: \operatorname{Im} Q \rightarrow \operatorname{KerL}$ such that $J\left(\left[\begin{array}{l}x \\ y\end{array}\right],\left[\begin{array}{l}0 \\ 0\end{array}\right], \cdots,\left[\begin{array}{l}0 \\ 0\end{array}\right]\right)=\left[\begin{array}{l}x \\ y\end{array}\right]$.

Define the homotopy $H_{v}=v(J Q N)+(1-v) G$ where

$$
G\left(\left[\begin{array}{l}
x \\
y
\end{array}\right]\right)=\left[\begin{array}{c}
\int_{0}^{w} a(s)-b(s) \exp (x) \Delta s+\ln \prod_{i=1}^{q}\left(1+g_{i}\right) \\
\int_{0}^{w} d(s)-\frac{f(s) \exp (x)}{\alpha(s)+\beta(s) \exp (x)+m(s) \exp (y)} \Delta s+\ln \prod_{i=1}^{q}\left(p_{i}\right)
\end{array}\right]
$$


Take $D J_{G}$ as the determinant of the jacobian of $G$. Since $\left[\begin{array}{l}x \\ y\end{array}\right] \in \operatorname{KerL}$, then jacobian of $G$ is

$$
\left[\begin{array}{cc}
-\mathrm{e}^{x} \int_{0}^{w} b(s) \Delta s & 0 \\
\int_{0}^{w} \frac{-\mathrm{e}^{x} f(s)}{\alpha(s)+\beta(s) \mathrm{e}^{x}+m(s) \mathrm{e}^{y}} \Delta s+\int_{0}^{w} \frac{\left(\mathrm{e}^{x}\right)^{2} f(s) \beta(s)}{\left(\alpha(s)+\beta(s) \mathrm{e}^{x}+m(s) \mathrm{e}^{y}\right)^{2}} \Delta s & -\int_{0}^{w} \frac{\mathrm{e}^{x} \mathrm{e}^{y} f(s) m(s)}{\left(\alpha(s)+\beta(s) \mathrm{e}^{x}+m(s) \mathrm{e}^{y}\right)^{2}} \Delta s
\end{array}\right] .
$$

All the functions in jacobian of $G$ is positive then $\operatorname{signDJ}_{G}$ is always positive. Hence

$$
\operatorname{deg}(J Q N, \Omega \bigcap \operatorname{KerL}, 0)=\operatorname{deg}(G, \Omega \cap \operatorname{KerL}, 0)=\sum_{\left[\begin{array}{l}
x \\
y
\end{array}\right] \in G^{-1}\left(\left[\begin{array}{l}
0 \\
0
\end{array}\right]\right)} \operatorname{signDJ}_{G}\left(\left[\begin{array}{l}
x \\
y
\end{array}\right]\right) \neq 0 .
$$

Thus all the conditions of Theorem 1 are satisfied. Therefore system (1) has at least a positive w-periodic solution.

Theorem 3. If same conditions are valid for the coefficient functions in system (1) and

$$
\begin{aligned}
& \left(\frac{\int_{0}^{w} a(t) \Delta t+\ln \prod_{i=1}^{q}\left(1+g_{i}\right)}{\int_{0}^{w} b(t) \Delta t}\right) \exp \left[-\left(2 \int_{0}^{w} a(t) \Delta t+\ln \prod_{i=1}^{q}\left(1+g_{i}\right)\right)\right] \\
& \cdot\left(\int_{0}^{w} f(t) \Delta t-\beta^{u}\left(\int_{0}^{w} d(t) \Delta t-\ln \prod_{i=1}^{q}\left(p_{i}\right)\right)\right)-\alpha^{u}\left(\int_{0}^{w} d(t) \Delta t-\ln \prod_{i=1}^{q}\left(p_{i}\right)\right)>0
\end{aligned}
$$

is satisfied then there exist at least a w-periodic solution.

Proof. First part of the proof is very similar with the proof of Theorem 2. By (2), (3) and (6)

$$
\int_{0}^{w} d(t) \Delta t-\ln \prod_{i=1}^{q}\left(p_{i}\right)=\int_{0}^{w} \frac{f(t) \exp (x(t))}{\alpha(t)+\beta(t) \exp (x(t))+m(t) \exp (y(t))} \Delta t \leq \exp \left(x\left(\eta_{1}\right)\right) \int_{0}^{w} \frac{f(t)}{\alpha(t)} \Delta t
$$

By (3) $\int_{0}^{w} d(t) \Delta t-\ln \prod_{i=1}^{q}\left(p_{i}\right)>0$. Also by the assumption of Theorem $3 f(t), \alpha(t)>0$. Then we get $x\left(\eta_{1}\right) \geq \tilde{l}_{1}, \quad \tilde{l}_{1}:=\ln \left(\frac{\int_{0}^{w} d(t) \Delta t-\ln \prod_{i=1}^{q}\left(p_{i}\right)}{\int_{0}^{w} f(t) / \alpha(t) \Delta t}\right)$.

And using the second inequality in Lemma 1 we have

$$
\begin{aligned}
x(t) & \geq x\left(\eta_{1}\right)-\int_{0}^{w}\left|x^{\Delta}(t)\right| \Delta t \\
& \geq x\left(\eta_{1}\right)-\left(\int_{0}^{w}|a(t)| \Delta t+\int_{0}^{w} a(t) \Delta t+\ln \prod_{i=1}^{q}\left(1+g_{i}\right)\right) \\
& =\tilde{H}_{1}:=\tilde{l}_{1}-\tilde{M}_{1} .
\end{aligned}
$$

By the first equation of (3) and (6)

$$
\int_{0}^{w} a(t) \Delta t+\ln \prod_{i=1}^{q}\left(1+g_{i}\right) \geq \int_{0}^{w} b(t) \exp \left(x\left(\xi_{1}\right)\right) \Delta t=\exp \left(x\left(\xi_{1}\right)\right) \int_{0}^{w} b(t) \Delta t
$$

Then we get $x\left(\xi_{1}\right) \leq \tilde{l}_{2}$ where $\tilde{l}_{2}:=\ln \left(\frac{\int_{0}^{w} a(t) \Delta t+\ln \prod_{i=1}^{q}\left(1+g_{i}\right)}{\int_{0}^{w} b(t) \Delta t}\right)$. 
Using the first inequality in Lemma 1 we have

$$
\begin{aligned}
x(t) & \leq x\left(\xi_{1}\right)+\int_{0}^{w}\left|x^{\Delta}(t)\right| \Delta t \\
& \leq x\left(\xi_{1}\right)+\left(\int_{0}^{w}|a(t)| \Delta t+\int_{0}^{w} a(t) \Delta t+\ln \prod_{i=1}^{q}\left(1+g_{i}\right)\right) \\
& \leq \tilde{H}_{2}:=\tilde{l}_{2}+\tilde{M}_{1} .
\end{aligned}
$$

By (12) and (13) $\max _{t \in[0, w]}|x(t)| \leq \tilde{B}_{1}:=\max \left\{\left|\tilde{H}_{1}\right|,\left|\tilde{H}_{2}\right|\right\}$. From the second equation of (3) and the second equation of (7), we can derive that

$$
\begin{aligned}
\int_{0}^{w} d(t) \Delta t-\ln \prod_{i=1}^{q}\left(p_{i}\right) & \leq \int_{0}^{w} \frac{f(t) \exp (x(t))}{m(t) \exp (y(t))} \Delta t \leq \int_{0}^{w} \frac{f(t) \mathrm{e}^{\tilde{H}_{2}}}{m(t) \exp \left(y\left(\xi_{2}\right)\right)} \Delta t \\
& =\frac{\mathrm{e}^{\tilde{H}_{2}}}{\exp \left(y\left(\xi_{2}\right)\right)} \int_{0}^{w} f(t) / m(t) \Delta t .
\end{aligned}
$$

Therefore

$$
\exp \left(y\left(\xi_{2}\right)\right) \leq \mathrm{e}^{\tilde{H}_{2}}\left(\frac{\int_{0}^{w} f(t) / m(t) \Delta t}{\int_{0}^{w} d(t) \Delta t-\ln \prod_{i=1}^{q}\left(p_{i}\right)}\right.
$$

Since $\mathrm{e}^{\tilde{H}_{2}}, f(t), m(t)>0$, then $y\left(\xi_{2}\right) \leq \tilde{L}_{1}$, where $\tilde{L}_{1}:=\ln \left(\mathrm{e}^{\tilde{H}_{2}}\left(\frac{\int_{0}^{w} f(t) / m(t) \Delta t}{\int_{0}^{w} d(t) \Delta t-\ln \prod_{i=1}^{q}\left(p_{i}\right)}\right)\right)$.

Hence, by using the first inequality in Lemma 1 and the second equation of (3),

$$
\begin{aligned}
y(t) & \leq y\left(\xi_{2}\right)+\int_{0}^{w}\left|y^{\Delta}(t)\right| \Delta t \\
& \leq y\left(\xi_{2}\right)+\left(\int_{0}^{w}|d(t)| \Delta t+\int_{0}^{w} d(t) \Delta t-\ln \prod_{i=1}^{q}\left(p_{i}\right)\right) \\
& \leq \tilde{H}_{3}:=\tilde{L}_{1}+\tilde{M}_{2} .
\end{aligned}
$$

By the assumption of Theorem 3 there exists $n_{0}$ such that $\forall n \geq n_{0}$

$$
\begin{aligned}
& \left(\frac{\int_{0}^{w} a(t) \Delta t+\ln \prod_{i=1}^{q}\left(1+g_{i}\right)}{\int_{0}^{w} b(t) \Delta t+1 / n \int_{0}^{w} c(t) / \alpha(t) \Delta t}\right) \exp \left[-\left(\int_{0}^{w}|a(t)| \Delta t+\int_{0}^{w} a(t) \Delta t+\ln \prod_{i=1}^{q}\left(1+g_{i}\right)\right)\right] \\
& \cdot\left(\int_{0}^{w} f(t) \Delta t-\beta^{u}\left(\int_{0}^{w} d(t) \Delta t-\ln \prod_{i=1}^{q}\left(p_{i}\right)\right)\right)-\alpha^{u}\left(\int_{0}^{w} d(t) \Delta t-\ln \prod_{i=1}^{q}\left(p_{i}\right)\right)>0
\end{aligned}
$$

is true. We need to get $\tilde{H}_{4}$ such that $\forall t \in[0, w]_{\mathbb{T}} \quad y(t) \geq \tilde{H}_{4}$. Let us assume there exists $t, s \in[0, w]_{\mathbb{T}}$ such that $y(s) \geq x(t)-\ln \left(n_{0}\right)$ Then by using (6) and (7) we obtain

$$
y\left(\eta_{2}\right) \geq y(s) \geq x(t)-\ln \left(n_{0}\right) \geq x\left(\xi_{1}\right)-\ln \left(n_{0}\right) \geq \tilde{H}_{1}-\ln \left(n_{0}\right):=M_{4}^{1} .
$$

If such $t$, $s$ does not exists then $\forall t, s \in[0, w]_{\mathbb{T}}, \quad y(s)<x(t)-\ln \left(n_{0}\right)$. Also from the first equation of (3), we have 


$$
\begin{aligned}
\int_{0}^{w} a(t) \Delta t+\ln \prod_{i=1}^{q}\left(1+g_{i}\right) & \leq \exp \left(x\left(\eta_{1}\right)\right) \int_{0}^{w} b(t) \Delta t+\exp \left(y\left(\eta_{2}\right)\right) \int_{0}^{w} \frac{c(t)}{\alpha(t)} \Delta t \\
& \leq \exp \left(x\left(\eta_{1}\right)\right)\left(\int_{0}^{w} b(t) \Delta t+\left(1 / n_{0}\right) \int_{0}^{w} \frac{c(t)}{\alpha(t)} \Delta t\right) .
\end{aligned}
$$

By using first inequality in Lemma 1 , we have $\exp (x(t)) \geq K$, where

$$
K:=\frac{\int_{0}^{w} a(t) \Delta t+\ln \prod_{i=1}^{q}\left(1+g_{i}\right)}{\left(\int_{0}^{w} b(t) \Delta t+\left(1 / n_{0}\right) \int_{0}^{w} \frac{c(t)}{\alpha(t)} \Delta t\right)} \exp -\left(\int_{0}^{w}|a(t)| \Delta t+\int_{0}^{w} a(t) \Delta t+\ln \prod_{i=1}^{q}\left(1+g_{i}\right)\right) .
$$

Using the second equality in (3) and the assumption of the Theorem 4, we obtain

$$
\int_{0}^{w} d(t) \Delta t-\ln \prod_{i=1}^{q}\left(p_{i}\right) \geq \frac{K}{\alpha^{u}+\beta^{u} K+m^{u} \exp \left(y\left(\eta_{2}\right)\right)} \int_{0}^{w} f(t) \Delta t
$$

This implies $y\left(\eta_{2}\right) \geq M_{4}^{2}$, where

$$
\begin{aligned}
M_{4}^{2}:= & \ln \left[\left(\frac{\int_{0}^{w} a(t) \Delta t+\ln \prod_{i=1}^{q}\left(1+g_{i}\right)}{\int_{0}^{w} b(t) \Delta t+\left(1 / n_{0}\right) \int_{0}^{w} c(t) / \alpha(t) \Delta t}\right) \exp \left[-\left(\int_{0}^{w}|a(t)| \Delta t+\int_{0}^{w} a(t) \Delta t+\ln \prod_{i=1}^{q}\left(1+g_{i}\right)\right)\right]\right. \\
& \left.\cdot\left(\int_{0}^{w} f(t) \Delta t-\beta^{u}\left(\int_{0}^{w} d(t) \Delta t-\ln \prod_{i=1}^{q}\left(p_{i}\right)\right)\right)-\alpha^{u}\left(\int_{0}^{w} d(t) \Delta t-\ln \prod_{i=1}^{q}\left(p_{i}\right)\right)\right] \\
& -\ln \left(m^{u}\left(\int_{0}^{w} d(t) \Delta t-\ln \prod_{i=1}^{q}\left(p_{i}\right)\right)\right) .
\end{aligned}
$$

Hence, according to the above discussion we have $y\left(\eta_{2}\right) \geq M_{4}:=\min \left\{M_{4}^{1}, M_{4}^{2}\right\}$. Using second inequality in Lemma 1 we have $y(t) \leq \tilde{H}_{4}$ where $\tilde{H}_{4}:=M_{4}-\left(\int_{0}^{w}|d(t)| \Delta t+\int_{0}^{w} d(t) \Delta t-\ln \prod_{i=1}^{q}\left(p_{i}\right)\right)$.

Thus $\max _{t \in[0, w]_{\mathbb{T}}}|y(t)| \leq \tilde{B}_{2}:=\max \left\{\left|\tilde{H}_{3}\right|,\left|\tilde{H}_{4}\right|\right\}$. Obviously, $\tilde{B}_{1}$ and $\tilde{B}_{2}$ are both independent of $\lambda$. Let $M=\tilde{B}_{1}+\tilde{B}_{2}+1$. Then $\max _{t \in[0, w]}\left\|\left[\begin{array}{l}x \\ y\end{array}\right]\right\|<M$. Let $\Omega=\left\{\left\|\left[\begin{array}{l}x \\ y\end{array}\right]\right\| \in X:\left\|\left[\begin{array}{c}x \\ y\end{array}\right]\right\|<M\right\}$ then $\Omega$ verifies the requirement (a) in Theorem 1. Rest of the proof is similar to Theorem 2.

Let there are two insect populations (one of them the predator, the other one the prey) both continuous while in season (say during the six warm months of the year), die out in (say) winter, while their eggs are incubating or dormant, and then both hatch in a new season, both of them giving rise to nonoverlapping populations. This situation can be modelled using the time scale

$$
\mathbb{T}=\bigcup_{k \in \mathbb{Z}}[2 k, 2 k+1], \quad \text { with } \omega=1
$$

Here impulsive effect of the pest population density is after its partial destruction by catching, poisoning with chemicals used in agriculture (can be shown by $-1<g_{k}<0$ ) and impulsive increase of the predator population density is by artificially breeding the species or releasing some species $\left(p_{k}>0\right)$. In addition to these, if the model assumes a BeddingtonDeAngelis functional response as in (1) and if the assumptions in Theorem 2 or 3 
are satisfied then there exists a 1-periodic solution of (1).

Corollary 1. If $\alpha(t)=0$ in the system (1) and

$$
\int_{0}^{w} f(t) \Delta t-\beta^{u}\left(\int_{0}^{w} d(t) \Delta t-\ln \prod_{i=1}^{q}\left(p_{i}\right)\right)>0
$$

is satisfied then the system (1) has at least one w-periodic solution.

Example 1. $\mathbb{T}=[2 k, 2 k+1], k \in \mathbb{N} k$ start with 0 .

$$
\begin{aligned}
x^{\Delta}(t)= & (0.2 \sin (2 \pi t)+0.3)-(0.2 \sin (2 \pi t)+0.2) \exp (x) \\
& -\frac{(0.1+0.1 \cos (2 \pi t)) \exp (y)}{(0.5 \sin (2 \pi t)+0.7)+(1+0.5 \cos (2 \pi t)) \exp (x)+\exp (y)}, t \neq t_{k} \\
y^{\Delta}(t)= & -(0.3 \sin (2 \pi t)+1)+\frac{(4 \cos (2 \pi t)+6.5) \exp (x)}{(0.5 \sin (2 \pi t)+0.7)+(1+0.5 \cos (2 \pi t)) \exp (x)+\exp (y)}, t \neq t_{k} \\
\Delta x\left(t_{k}\right)= & \ln \left(1+g_{k}\right) \\
\Delta y\left(t_{k}\right)= & \ln \left(p_{k}\right)
\end{aligned}
$$

Impulse points: $t_{1}=2 k+1 / 4, t_{2}=2 k+3 / 4$ and $q=2$.

$g_{1}=\mathrm{e}^{-0.01}-1, g_{2}=\mathrm{e}^{-0.01}-1$

$p_{1}=\mathrm{e}^{0.1}, \quad p_{2}=\mathrm{e}^{0.1}$

Example 1 satisfies all the conditions of Theorem 2, thus it has at least one periodic solution.

Example 2. $\mathbb{T}=[2 k, 2 k+1], k \in \mathbb{N} \quad k$ start with 0 .

$$
\begin{aligned}
x^{\Delta}(t)= & (0.2 \sin (2 \pi t)+0.3)-(0.1 \sin (2 \pi t)+0.2) \exp (x) \\
& -\frac{(3+\cos (2 \pi t)) \exp (y)}{(\sin (2 \pi t)+2)+(1+0.5 \cos (2 \pi t)) \exp (x)+1.5 \exp (y)}, t \neq t_{k} \\
y^{\Delta}(t)= & -(0.3 \sin (2 \pi t)+1)+\frac{(4 \cos (2 \pi t)+6.5) \exp (x)}{(\sin (2 \pi t)+2)+(1+0.5 \cos (2 \pi t)) \exp (x)+1.5 \exp (y)}, t \neq t_{k} \\
\Delta x\left(t_{k}\right)= & \ln \left(1+g_{k}\right) \\
\Delta y\left(t_{k}\right)= & \ln \left(p_{k}\right)
\end{aligned}
$$

Impulse points: $t_{1}=2 k+1 / 4, t_{2}=2 k+3 / 4$ and $q=2$.

$g_{1}=\mathrm{e}^{-0.01}-1, g_{2}=\mathrm{e}^{-0.01}-1$

$p_{1}=\mathrm{e}^{0.1}, \quad p_{2}=\mathrm{e}^{0.1}$

Example 2 satisfies all the conditions of Theorem 3, thus it has at least one periodic solution.

Theorem 4. If all the coefficient functions in system (1) is positive, w-periodic, from $C_{r d}\left(\mathbb{T}, \mathbb{R}^{2}\right)$ and impulses are 0; also

$$
\begin{aligned}
& \left(\frac{a^{l}}{b^{u}}\right) \exp \left(\mu\left(a^{l}-b^{u}\left(a^{u} / b^{l}\left(\exp \left(\mu a^{u}\right)\right)\right)-c^{u} / m^{l}\right)\right) \\
& \cdot\left(\int_{0}^{w} f(t) \Delta t-\beta^{u}\left(\int_{0}^{w} d(t) \Delta t\right)\right)-\alpha^{u}\left(\int_{0}^{w} d(t) \Delta t\right)>0
\end{aligned}
$$

is satisfied then there exist at least a w-periodic solution. $\mu=\max _{[0, w]_{\mathbb{T}}} \mu(t)$

Proof. First part of the proof is similar to Theorem 2, only difference is the zero impulses. If the assumption of Theorem 4 is true then there exists $n_{0}$ such that for all $n \geq n_{0}$ 


$$
\begin{aligned}
& \left(\frac{a^{l}}{b^{u}}+\left(1 / n_{0}\right) \frac{c^{u}}{a^{l}}\right) \exp \left(\mu\left(a^{l}-b^{u}\left(a^{u} / b^{l}\left(\exp \left(\mu a^{u}\right)\right)\right)-c^{u} / m^{l}\right)\right) \\
& \cdot\left(\int_{0}^{w} f(t) \Delta t-\beta^{u}\left(\int_{0}^{w} d(t) \Delta t\right)\right)-\alpha^{u}\left(\int_{0}^{w} d(t) \Delta t\right)>0
\end{aligned}
$$

is satisfied. Suppose there exist $s, t \in[0, w]_{\mathbb{T}}$ such that $y(s) \geq x(t)-\ln \left(n_{0}\right)$. Then similar to proof of Theorem 4 we can find $\hat{M}_{4}^{1}$.

If such $s, t$ does not exist $y(s)<x(t)-\ln \left(n_{0}\right)$. Using the first equation of (1) and assuming $\sigma(\tilde{t})$ is the minimum of $x(t)$. Then

$$
0 \geq(x(\tilde{t}))^{\Delta}=a(\tilde{t})-b(\tilde{t}) \exp (x(\tilde{t}))-\frac{c(\tilde{t}) \exp (y(\tilde{t}))}{\alpha(\tilde{t})+\beta(\tilde{t}) \exp (x(\tilde{t}))+m(\tilde{t}) \exp (y(\tilde{t}))} .
$$

Thus we get

$$
a^{l} \leq b^{u} \exp (x(\tilde{t}))+\frac{c^{u} \exp (y(\tilde{t}))}{\alpha^{l}} \leq\left(b^{u}+\left(1 / n_{0}\right) c^{u} / \alpha^{l}\right) \exp (x(\tilde{t}))
$$

Then $\exp (x(\tilde{t})) \geq \frac{a^{l}}{b^{u}+\left(1 / n_{0}\right) c^{u} / \alpha^{l}}$.

If $\tilde{t}$ is a right dense point then $\exp (x(\sigma(\tilde{t}))) \geq \frac{a^{l}}{b^{u}+\left(1 / n_{0}\right) c^{u} / \alpha^{l}}$. If $\tilde{t}$ is right scattered, we interested with the maximum of the solution. Let $\sigma(\hat{t})$ be the maximum of $x(t)$.

$$
0 \leq(x(\hat{t}))^{\Delta}=a(\hat{t})-b(\hat{t}) \exp (x(\hat{t}))-\frac{c(\hat{t}) \exp (y(\hat{t}))}{\alpha(\hat{t})+\beta(\hat{t}) \exp (x(\hat{t}))+m(\hat{t}) \exp (y(\hat{t}))} \leq a^{u} .
$$

Then $\exp (x(\hat{t})) \leq a^{u} / b^{l}$. If $\hat{t}=\sigma(\hat{t})$, then $\exp (x(\sigma(\tilde{t}))) \leq a^{u} / b^{l}$.

If $\hat{t} \neq \sigma(\hat{t})$, then $\exp (x(\sigma(\hat{t}))) \leq a^{u} / b^{l}\left(\exp \left(\mu a^{u}\right)\right)$.

Thus

$$
\exp (x(\sigma(\tilde{t}))) \geq \frac{a^{l}}{b^{u}+\left(1 / n_{0}\right) c^{u} / \alpha^{l}} \exp \left(\mu\left(a^{l}-b^{u}\left(a^{u} / b^{l}\left(\exp \left(\mu a^{u}\right)\right)\right)-c^{u} / m^{l}\right)\right)=K_{1} .
$$

Using (3) and (7) above results we obtain

$$
\int_{0}^{w} d(t) \Delta t \geq \frac{K_{1}}{\alpha^{u}+\beta^{u} K_{1}+m^{u} \exp \left(y\left(\eta_{2}\right)\right)} \int_{0}^{w} f(t) \Delta t
$$

This implies

$$
\begin{aligned}
& y\left(\eta_{2}\right) \geq \ln {\left[\left(\frac{a^{l}}{b^{u}+\left(1 / n_{0}\right) c^{u} / \alpha^{l}} \exp \left(\mu\left(a^{l}-b^{u}\left(a^{u} / b^{l}\left(\exp \left(\mu a^{u}\right)\right)\right)-c^{u} / m^{l}\right)\right)\right)\right.} \\
&\left.\cdot\left(\int_{0}^{w} f(t) \Delta t-\beta^{u} \int_{0}^{w} d(t) \Delta t\right)-\alpha^{u}\left(\int_{0}^{w} d(t) \Delta t\right)\right]-\ln \left(m^{u}\left(\int_{0}^{w} d(t) \Delta t\right)\right)=\hat{M}_{4}^{2} .
\end{aligned}
$$

Hence, according to the above discussion we have $y\left(\eta_{2}\right) \geq \hat{M}_{4}=\min \left\{\hat{M}_{4}^{1}, \hat{M}_{4}^{2}\right\}$. Using second inequality in Lemma 1 we have $y(t) \leq \hat{M}_{4}-\left(2 \int_{0}^{w} d(t) \Delta t\right)=\hat{H}_{4}$. Thus $\max _{t \in[0, w]_{\mathbb{T}}}|y(t)| \leq \max \left\{\left|\tilde{H}_{3}\right|,\left|\hat{H}_{4}\right|\right\}$. Rest of the proof 
is similar to Theorem 2 .

Corollary 2. In Theorem 4 if we take $\mathbb{T}$ as $\mathbb{R}$ then we get Theorem 3 in [21].

Example 3. $\mathbb{T}=[2 k, 2 k+1], k \in \mathbb{N} \quad k$ start with 0 .

$$
\begin{aligned}
x^{\Delta}(t)= & (0.1 \sin (2 \pi t)+0.2)-(0.1 \sin (2 \pi t)+0.25) \exp (x) \\
& -\frac{(0.1+0.1 \cos (2 \pi t)) \exp (y)}{(0.2 \sin (2 \pi t)+0.2)+(1+0.5 \cos (2 \pi t)) \exp (x)+\exp (y)} \\
y^{\Delta}(t)= & -(0.3 \sin (2 \pi t)+1)+\frac{(4 \cos (2 \pi t)+6.5) \exp (x)}{(0.2 \sin (2 \pi t)+0.2)+(1+0.5 \cos (2 \pi t)) \exp (x)+\exp (y)}
\end{aligned}
$$

Example 3 satisfies all the conditions of Theorem 4, thus it has at least one periodic solution.

All the graphs that we see in Figures 1-3 are obtained by Mathlab.

\section{Discussion}

In this paper, the impulsive predator-prey dynamic systems on time scales calculus are studied. We investigate when the system has periodic solution. Furthermore, three different conditions have been found which are necessary for the periodic solution of the predator-prey dynamic systems with Beddington-DeAngelis type functional response. Also by using graphs, we are able to show that the conditions that are found in Theorem 2, 3
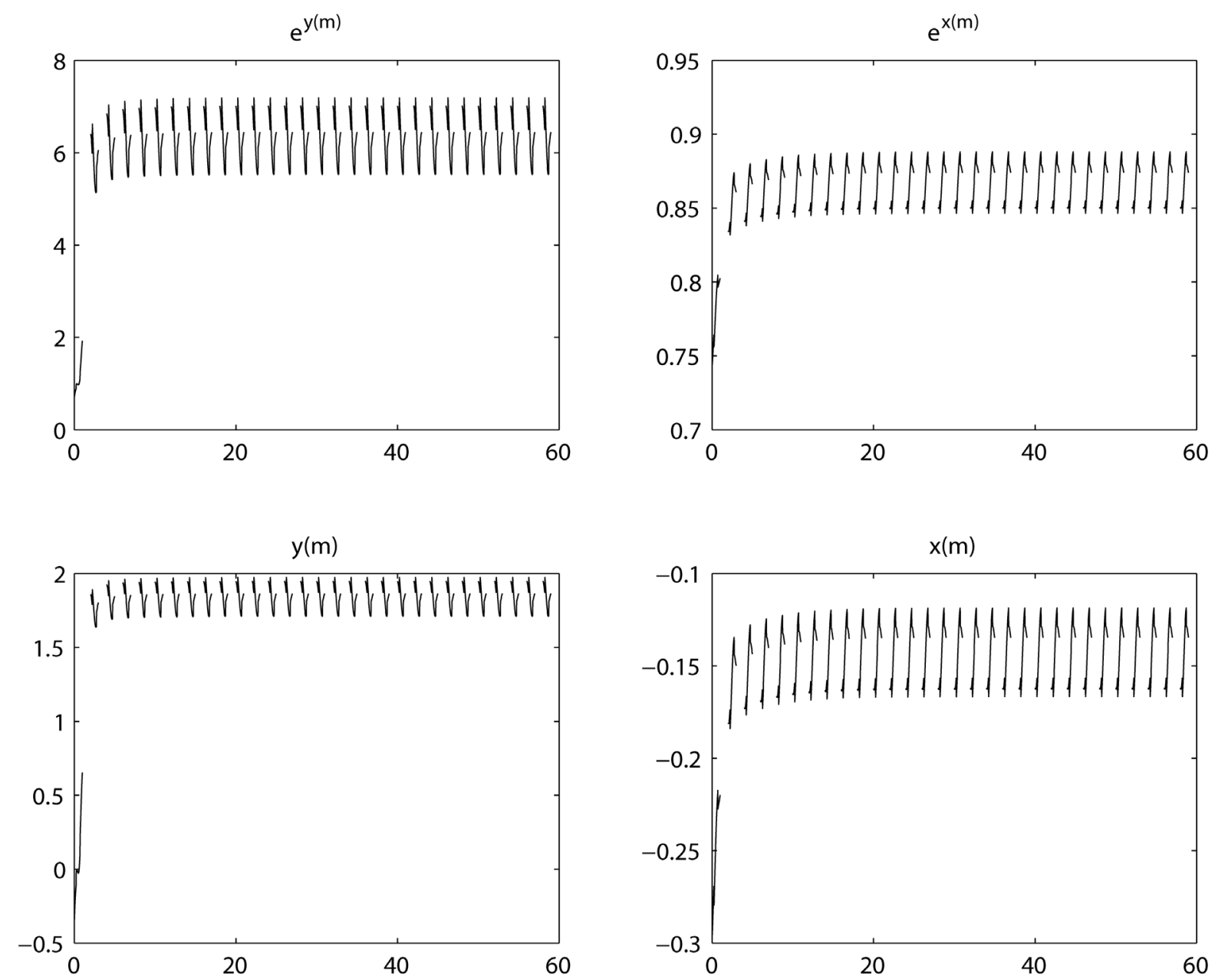

Figure 1. Numeric solution of Example 1 shows the periodicity. 

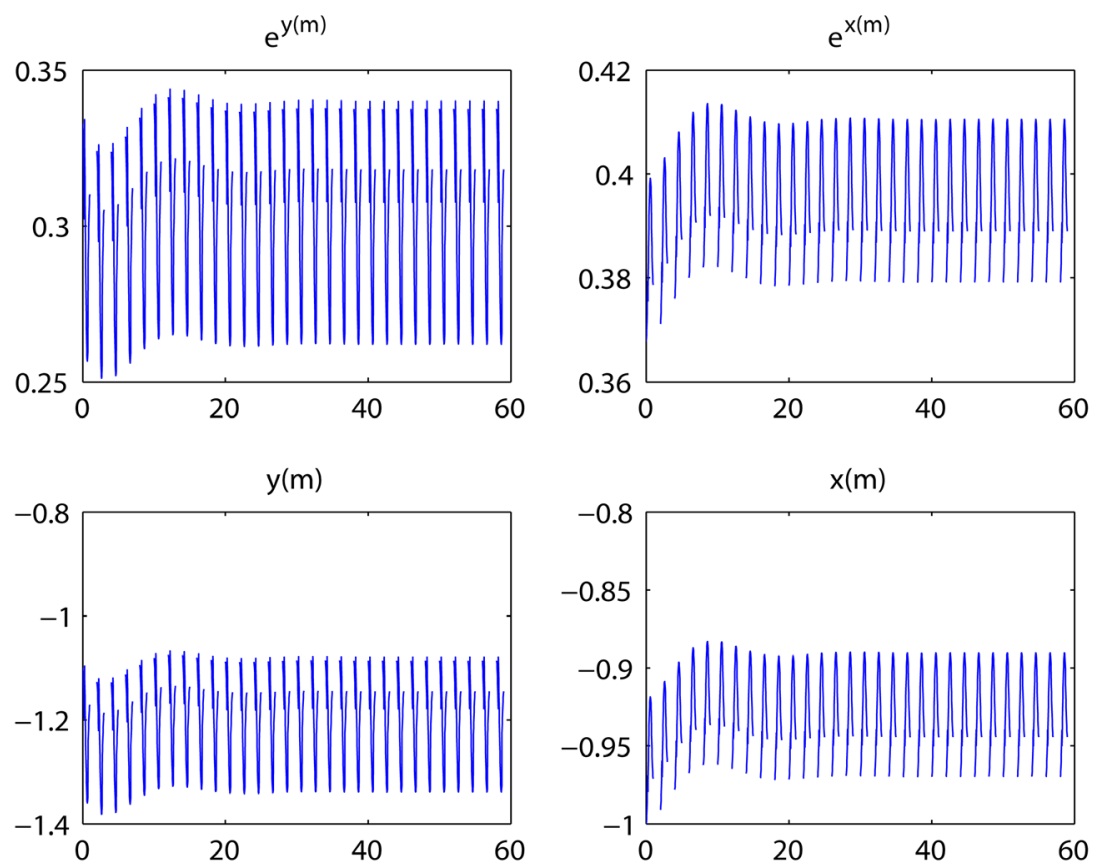

Figure 2. Numeric solution of Example 2 shows the periodicity.
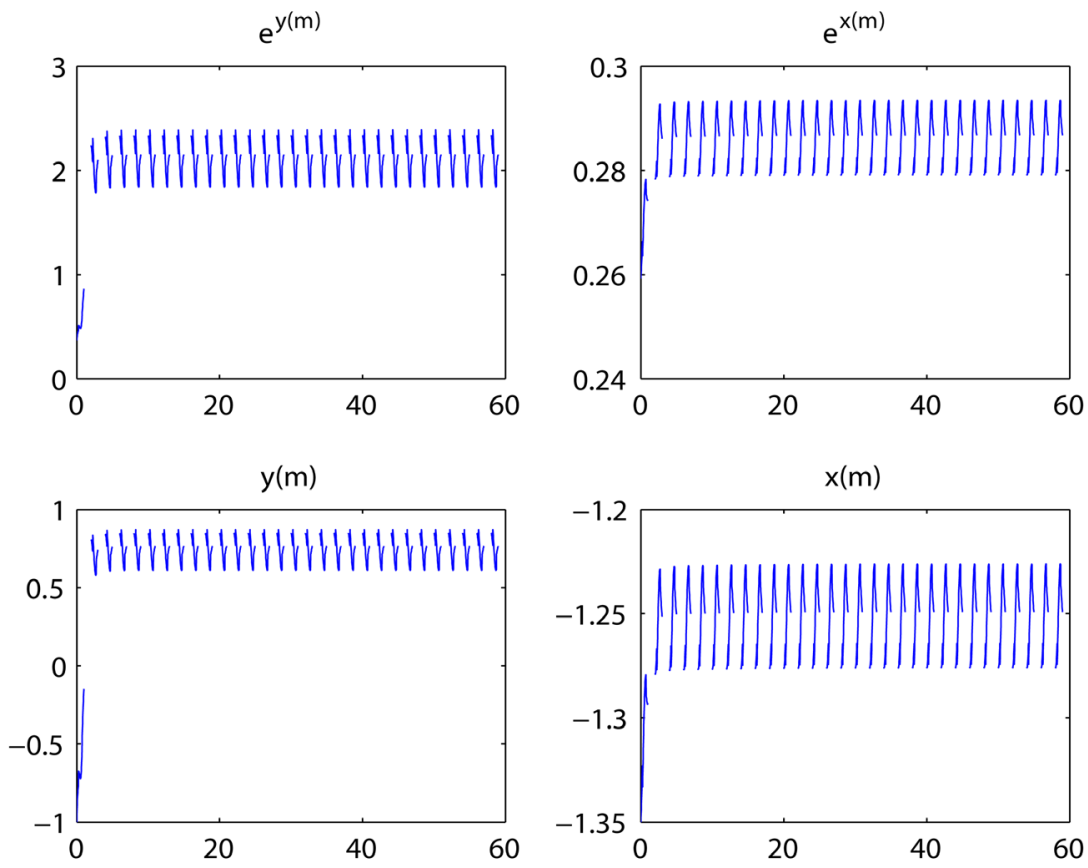

Figure 3. Numeric solution of Example 3 shows the periodicity.

and 4 are enough for the periodic solution of the given system. In this work, since our system can model the life cycle of the such species like insects, what we have done new is finding necessary condition for the periodic solution of the given predator-prey system with sudden changes. In addition to these, according to the structure of the given time scale $\mathbb{T}$, the conditions that are found in Theorem 2, 3 and 4 become useful.

\section{References}

[1] Wang, W., Shen, J. and Nieto, J. (2007) Permanence and Periodic Solution of Predator-Prey System with Holling Type 
Functional Response and Impulses. Discrete Dynamics in Nature and Society, 2007, Article ID: 81756, 15 p.

[2] Beddington, J.R. (1975) Mutual Interference between Parasites or Predators and Its Effect on Searching Efficiency. Journal of Animal Ecology, 44, 331-340. http://dx.doi.org/10.2307/3866

[3] DeAngelis, D.L., Goldstein, R.A. and O’Neill, R.V. (1975) A Model for Trophic Interaction. Ecology, 56, $881-892$. http://dx.doi.org/10.2307/1936298

[4] Fan, M. and Agarwal, S. (2002) Periodic Solutions for a Class of Discrete Time Competition Systems. Nonlinear Studies, 9, 249-261.

[5] Fan, M. and Wang, K. (2000) Global Periodic Solutions of a Generalized n-Species GilpinAyala Competition Model. Computers \& Mathematics with Applications, 40, 1141-1151. http://dx.doi.org/10.1016/S0898-1221(00)00228-5

[6] Fan, M. and Wang, K. (2001) Periodicity in a Delayed Ratio-Dependent Predatorprey System. Journal of Mathematical Analysis and Applications, 262, 179-190. http://dx.doi.org/10.1006/jmaa.2001.7555

[7] Fan, M. and Wang, Q. (2004) Periodic Solutions of a Class of Nonautonomous Discrete Time Semi-Ratio-Dependent Predatorprey Systems. Discrete and Continuous Dynamical Systems, Series B, 4, 563-574. http://dx.doi.org/10.3934/dcdsb.2004.4.563

[8] Fang, Q., Li, X. and Cao, M. (2012) Dynamics of a Discrete Predator-Prey System with Beddington-DeAngelis Function Response. Applied Mathematics, 3, 389-394. http://dx.doi.org/10.4236/am.2012.34060

[9] Huo, H.F. (2005) Periodic Solutions for a Semi-Ratio-Dependent Predatorprey System with Functional Responses. Applied Mathematics Letters, 18, 313-320. http://dx.doi.org/10.1016/j.aml.2004.07.021

[10] Li, Y.K. (1999) Periodic Solutions of a Periodic Delay Predatorprey System. Proceedings of the American Mathematical Society, 127, 1331-1335. http://dx.doi.org/10.1090/S0002-9939-99-05210-7

[11] Wang, Q., Fan, M. and Wang, K. (2003) Dynamics of a Class of Nonautonomous Semi-Ratio-Dependent PredatorPrey Systems with Functional Responses. Journal of Mathematical Analysis and Applications, 278, 443-471. http://dx.doi.org/10.1016/S0022-247X(02)00718-7

[12] Xu, R., Chaplain, M.A.J. and Davidson, F.A. (2005) Periodic Solutions for a Predato-Prey Model with Holling-Type Functional Response and Time Delays. Applied Mathematics and Computation, 161, 637-654. http://dx.doi.org/10.1016/j.amc.2003.12.054

[13] Bainov, D. and Simeonov, P. (1993) Impulsive Differential Equations: Periodic Solutions and Applications, Pitman Monographs and Surveys in Pure and Applied Mathematics. Vol. 66, Longman Scientific and Technical, Harlow.

[14] Samoilenko, A.M. and Perestyuk, N.A. (1995) Impulsive Differential Equations. World Scientific Series on Nonlinear Science. Series A: Monographs and Treatises, Vol. 14, World Scientific, River Edge.

[15] Lakshmikantham, V., Banov, D.D. and Simeonov, P.S. (1989) Theory of Impulsive Differential Equations. Series in Modern Applied Mathematics, Vol. 6, World Scientific, Teaneck. http://dx.doi.org/10.1142/0906

[16] Wang, P. (2006) Boundary Value Problems for First Order Impulsive Difference Equations. International Journal of Difference Equations, 1, 249-259.

[17] Tang, S., Xiao, Y., Chen, L. and Cheke, R.A. (2005) Integrated Pest Management Models and Their Dynamical Behaviour. Bulletin of Mathematical Biology, 67, 115-135. http://dx.doi.org/10.1016/j.bulm.2004.06.005

[18] Xiang, Z., Li, Y. and Song, X. (2009) Dynamic Analysis of a Pest Management SEI Model with Saturation Incidence Concerning Impulsive Control Strategy. Nonlinear Analysis, 10, 2335-2345. http://dx.doi.org/10.1016/j.nonrwa.2008.04.017

[19] Wei, C. and Chen, L. (2012) Periodic Solution of Prey-Predator Model with Beddington-DeAngelis Functional Response and Impulsive State Feedback Control. Journal of Applied Mathematics, 2012, Article ID: 607105. http://dx.doi.org/10.1155/2012/607105

[20] Bohner, M., Fan, M. and Zhang, J.M. (2006) Existence of Periodic Solutions in Predator-Prey and Competition Dynamic Systems. Nonlinear Analysis: Real World Applications, 7, 1193-1204. http://dx.doi.org/10.1016/j.nonrwa.2005.11.002

[21] Fazly, M. and Hesaaraki, M. (2008) Periodic Solutions for Predator-Prey Systems with Beddington DeAngelis-Functional Response on Time Scales. Nonlinear Analysis: Real World Applications, 9, 1224-1235. http://dx.doi.org/10.1016/j.nonrwa.2007.02.012 